\title{
Study on Current Situation of Geography Learning of Junior Middle School Students in Chuxiong City
}

\author{
Sumei Guo ${ }^{1, a}$ and Wujun $\mathrm{Xi}^{1, \mathrm{~b}}$ \\ ${ }^{1}$ School of Geography and Tourism Management, Chuxiong Normal University, Chuxiong, China \\ a1347999343@qq.com, babsxwj@163.com
}

Keywords: Junior school student; Geography learning; Chuxiong city

\begin{abstract}
To understand the status of junior middle school students geography learning in Chuxiong city, reduce blindness of geography teaching, improve teaching quality and efficiency, a geography learning questionnaire survey of four junior middle schools in Chuxiong city was made. Some problems were found as follows: some students lacked of interest in learning geography, positivity to geography learning was not enough, input amount of time and energy to geography learning was less, self -exploration sense was weak. So, the following countermeasures and suggestions were put forward, the teachers and students should cultivate the interest in geography learning, improve positivity to geography learning, change the geography learning methods, explore suitable ways of learning, strengthen communication between teachers and students, improve the student's self-exploration ability, allocate after-school time to geography learning reasonably.
\end{abstract}

\section{楚雄市城区初中生地理学习现状分析

\author{
国素梅 ${ }^{1, a}$, 席武俊 ${ }^{1, b}$ \\ 1. 楚雄师范学院地理科学与旅游管理学院, 中国云南楚雄市 675000
}

a1347999343@qq.com, babsxwj@163.com

摘要: 为了解楚雄市城区初中生地理学习现状, 减少地理教学的盲目性, 提高教学质量和教 学效率, 通过问卷调查对楚雄市城区 4 所中学初中生地理学习现状进行调查。发现初中生在 地理学习过程中存在的一些问题: 部分学生缺乏学习地理的兴趣; 地理学习的主动性、积极 性不高; 课外对地理学习的投入较少; 地理学习过程中自主探究意识薄弱。基于此提出相应 的对策及建议: 重视地理学习兴趣的培养, 提高地理学习的积极性; 改变地理学习方法, 探 求合适的学习方式; 师生间多沟通交流, 培养学生主动探究能力; 分配好课外地理学习时间, 构成课外学习时间的最优化。

关键词: 初中生; 地理学习; 楚雄市

\section{1. 引言}

在地理课程改革下, 初中地理课程标准中强调要关注学生作为 “整体的人” 的发展, 回归学 生的生活世界, 更好地突出学生的主体地位。现代地理教育的形态是教学, 不但包括教师的 教, 而且包括学生的学。教学研究不但要研究教师的教, 而且要研究学生的学。研究学生的 学, 不但要研究学习的外部表现形式, 更要研究学习的心理过程 [1]。而地理学科是基础教育、 素质教育的重要组成成分, 其自身具有一定的趣味性。学情分析是教学目标设定的基础, 是 分析教学内容的依据, 是教学方针选择和教学活动设计的落脚点 [2]。

在学习方法方面, 美国哈伯特 - 赛伦首次提及了学习方式, 也叫学习风格。西方学者从分歧 的视角把学习方式分为了不同的类别, 如沉思型与冲动型、场独立性与场依存型等 [3]。罗杰 斯在 20 世纪 60 年代就提到以学生为中心的教育理念, 并指出, 真正有意义的学习只有在所 学内容具备个人相关性和学习者能积极参与之时 [4]。在学习策略方面, 最具有代表性的是美 
国的帕金斯，他从学习情境的角度出发提出了有效迁移策略，包括了拥抱式策略、架桥式策 略两种策略 [5]。在国内有关地理学习方面的研究也是屡见不鲜。例如在地理学习兴趣研究方 面, 具有代表性的是孙尚福 [6]、谢月美 [7]、何智丰 [3]等。地理学习策略研究方面, 典型性 较强的是邵胜新 [8]。フ传芳和高如珊将众多的地理教学方式分成认知活动方式、行为活动方 式和地理课题讲授方式等三大组块 $[9]$ 。

对楚雄市城区初中生地理学习近况进行调查分析, 可以更好地了解初中生地理学习近况, 对 初中生地理学习方法进行较为合理的思考, 促使初中生更好地把握地理学习要领和地理研究 方法, 为构建高效课堂提供一些参考依据。

\section{2. 调查对象与方法}

\section{1. 调查对象}

在此次的调查中主要选取楚雄市城区的楚雄师院附中、天人中学初一年级各一个班, 楚雄一 中、紫溪中学初二年级各一个班进行调查。

2.2. 问卷制定

问卷来源于初中生地理学习现状调查问卷 ${ }^{[10]}$, 在此基础上进行了一定的修改。修改后的调查 问卷内容全部为单项选择题, 包括 2 个基本信息题, 主体部分为 19 个选择题, 涉及到的题目 有地理学习方式、地理教学方式、地理课外学习时间、查阅地理信息的主动性、查阅地理信 息的途径、课后讨论地理问题的情况、地理学习的目的等。

2.3. 调查过程

本次发放问卷 200 份, 回收 196 份, 回收率 97\%, 达到问卷发放与回收的标准。若问卷存在 部分或全数题目未填写, 则视为废卷。其中楚雄一中初二 (1) 班发放问卷 48 份, 回收 48 份, 回收率 100\%, 有效问卷 48 份, 有效率 100\%, 楚雄师院附中初一（113）班发放问卷 46 份, 回收 46 份, 回收率 100\%, 有效问卷 46 份, 有效率 100\%, 紫溪中学初二（2）班发放问卷 57 份, 回收 55 份, 回收率 96\%, 有效问卷 55 份, 有效率 100\%, 天人中学初一发放问卷 50 份, 回收 47 份，回收率 94\%，有效问卷 45 份，有效率 98\%。

\section{3. 楚雄市初中生地理学习现状问卷调查结果与分析}

\section{1. 个人基本信息}

四所学校中被调查的女生所占比例比男生大。整体来看，男生约占 40\%，女生约占 $60 \%$ 。

2.2. 问卷主体题目的结果分析

学生地理课喜爱程度方面, 对地理课喜爱程度为 “一般” 的比例最大, 占 $50.5 \%$, 程度为 “喜 欢” 占 $44 \%$, “不喜欢” 的占 $3.6 \%$, “不知道” 占 $1.9 \%$ 。说明地理作为一门趣味性学科, 还是 较受学生欢迎的。但另有极少数学生不喜欢地理课或者是不清楚, 对于这样的学生教师应该 着重培养和激发学生地理学习兴趣, 使学生体会到地理自身存在的趣味性, 以提高学生学习 地理的兴趣。

初中生课后用于地理学习的时间方面, 初中生在课外用于地理学习的时间为 “ $0-1$ 小时” 的 占 65\%, “1-2 小时” 的占 18\%, “从不花时间” 占 14. 5\%, “2 小时以上” 的占 $2.5 \%$ 。说明大多 数学生 (65\%) 对待地理这一学科还是比较认真的, 对于课后 “从不花时间” 学习地理的学生, 教师应当给予适当的引导，优化地理学习时间，合理利用地理学习时间。

在课堂上做演示实验的情况方面, 对于在课堂上做演示实验为 “比较希望” 所占比例最大, 达到 41. 1\%, “非常希望”占 38.1\%, “一般”占 14.3\%, “无所谓” 占 6.5\%。因为初中生好奇 心强, 多做演示实验不但可以增强兴趣, 还可以集中学生上课的注意力, 取得较好的学习效 果。

学生的地理学习方式方面, “多让学生合作交流, 自主探究” 的学习方式比例为 $71.5 \%$, 其次 是 “安静的听老师讲课” 占 $24.5 \%$, “老师少讲, 学生多做练习, 多做试卷” 的学习方式只占 
$4 \%$ 。由此可以看出, “多让学生合作交流, 自主探究” 的学习方式备受喜爱, 而之前较为死板 的学习方式已经不适合现在学生, 因此教师应该根据这些现象, 采取更为灵活的方式进行教 学。

学生答错问题时对老师态度的期望方面, 学生在答问错题时希望老师 “老师稍微提示, 让你 继续作答” 的占 $85.5 \%$, 希望老师 “不点评, 别人来回答” 和 “不知道” 的各自占 $6 \%$, 希望 “老师狠狠批评” 的占 $2.5 \%$ 。从结果中可以看出学生之间在心理上存在一定的差别, 看待事 物的立场和方式也就不一样, 因此教师应该根据学生各自的特点因材施教, 多了解学习者, 多和他们联系。

学生所喜爱课堂类型方面, 学生们都比较喜欢 “师生互动多, 气氛活跃” 的课堂, 所占比例 为 $84 \%$, 其次是 “讲授多, 知识量大” 的课堂占 7\%, “纪律要求严格” 的课堂占 4\%, “不知道” 的占 $5 \%$ 。针对这样的现象, 在教学过程中教师应该采取相应的措施, 多让学生进行互动、讨 论交流, 同时还应想方法激发学生学习的积极性, 保证学生都能参与到课堂中来, 让学生体 味学习的兴趣。

学生感兴趣的的地理信息查阅的主动性方面, “偶尔” 查阅地理信息的占 $70.8 \%$, “不会” 查 阅地理信息的占 $16.7 \%$, “常常” 查阅地理信息的占 $12.5 \%$ 。说明初中生的主动性还有些欠缺。 学生在课后讨论地理问题情况方面, 在课后讨论问题的情况为 “一般” 所占比例最高, 达到 了 $44 \%$, 其次是 “不经常” 占 $38.5 \%$, “从不” 占 $13.5 \%$; 所占比例最小的为 “常常”, 只占了 $4 \%$ 。这一结果表明, 在一定程度上有些学生仍然是缺少自主学习的意识, 主动性薄弱。因此, 在教学过程中教师应该多引导学生讨论问题, 大胆发表自己的意见, 提高学习的主动性, 培 养学生发现问题, 解决问题的习惯, 提高处理问题的能力。

学生上网查阅信息的路径方面, 通过 “搜索引擎” 查阅信息的占 $71.5 \%$; “直接在地址栏中输 入关键词” 查询的占 $22.8 \%$, 通过 “专题网站” 搜寻的占 $4 \%$, 通过 “门户网站” 查找的占 $1.7 \%$ 。 绝大部分学生喜欢采用 “搜索引擎” 的方式进行查阅信息是因为简单方便，易于操作。

初中生学习地理的最终目的方面, 学生认为学习地理的终究目标是 “用于生活” 的占 49\%, “掌 握地理规律” 占 $27 \%$, 认为学习地理只是为了 “记住地理常识” 的占 $20 \%$, “不清楚” 的占 $4 \%$ 。 这些现象在一定程度上反映了当代初中生学习地理的兴趣所在点, 地理比较贴近现实生活。 学生喜爱的教学方法方面, “老师引导, 学生合作探究” 的教学方式备受学生欢迎, 所占比例 为 77\%, 其次是 “老师布置任务, 学生讨论交流” 占了 13. 2\%; “老师讲, 学生安静地听” 的 教学方法占 5. 8\%, “老师布置任务, 学生自学” 的比例最小为 $4 \%$ 。显而易见, 学生们都比较 喜欢合作探究的上课方式, 合作探究可以增强学生之间的交流与合作, 可以轻松和愉快的获 取地理知识, 彰显学习着的主体地位。因此在课堂教学过程中, 教师应该更多注意引导学生, 以学生为主体, 鼓励学生合作探究, 不断提高学生学习的积极性和主动性。

画图对地理学习的帮助方面, 认为 “画图作用比较大” 的占 40\%, “非常大” 占 $26.8 \%$, “一般” 占 $26.2 \%$, “不清楚” 占 7\%, 大部分学生认为画图对地理学习帮助 “比较大”, 小部分则不清 楚。这些现象说明了多数初中生认为画图对地理学习是有帮助的, 因此在教学过程中教师应 该告诉学生画图的重要性, 画图是地理学习是最基础的东西, 在生活中好多地方都用到了地 图, 要求学生提高画图对地理学习重要性的意识。

地理知识对外出旅游的帮助方面, “用到很多” 占 $25 \%$, “一般” 占 $40.8 \%$, “一点” 占 $23.7 \%$, “完全没用到” 占 $10.5 \%$ 。这一定程度上说明, 地理知识与我们的生活紧紧联系在一起, 地 理源于生活, 正好符合了初中地理的基本理念之一: 学习对生活有用的地理。渐渐养成对地 理学习的好奇心及兴趣, 逐步养成求真务实的科学态度以及地理审美观, 进而增强地理学习 意识和积极性。

3. 3 . 小结

通过对楚雄市城区初中生地理学习现状调查分析, 得出的主要结论有: 部分学生缺乏学习地 理的兴趣, 对地理学习兴趣不浓厚; 学习地理的主动性、积极性不高; 课外对地理学习的投 入 (查阅资料、做课后作业时间、讨论地理问题) 较少; 地理学习过程当中自主探究意识较 
弱。

\section{4. 改善楚雄市城区初中生地理学习的对策与建议}

4.1. 重视地理学习兴趣的培养, 提高地理学习的积极性

针对楚雄市城区初中生地理学习兴趣欠缺情况, 老师在上课的时候要细心设计教学引言, 诱 发兴趣。按照课堂教学内容, 提出带有悬念的题目, 指引学者、启迪学生思索。除了教师对 学生的学习兴趣有影响外, 对于学生而言其本身的学习兴趣也是至关重要的。因此在地理学 习过程当中，学生应该主动培养学习兴趣。在业余时间可以看一些有关地理方面的电视、电 影, 读一些和地理有关的人物传纪等, 培养地理学习的兴趣爱好, 提高地理学习的积极性和 主动性。

针对问卷中学生出现的缺乏主动性的问题，教师应该开展一些实践性活动。开展实践性活动 可以让学生更好地感知地理事物, 明白学习源于生活的道理, 从而培养学习的兴趣, 养成良 好的学习习惯, 提高学习的积极主动性。在条件允许的情况下, 教师可以通过做一些演示实 验的方法来培养学生的学习兴趣, 调整学习心态。

4.2. 改变地理学习方法, 探求合适的学习方式

在地理学习中, 学生除了在教师指引下学习外, 同时该还应该注重本身的地理学习方式。学 生比较喜欢 “合作交流、探讨” 的学习方式, 因此, 教师应该从这方面引导学生。适合自身 的学习方法才是最好的学习方法, 在学习过程中学生应该不断摸索寻找出适合自己的学习方 法。学生应当按照学习内容的不同采取不一样的学习方法, 去除传统的死记硬背的学习方法, 找到适合自己的学习方法，提升学习效率。

4.3. 师生间多沟通交流, 培养学生主动探究能力

轻松活跃的课堂氛围是学生非常向往的, 因此, 在地理教学过程中, 教师应该观察课堂气氛, 尽量营造轻松活跃的课堂, 这样学生就可以在愉快的氛围中学到了新的知识。学生是教育的 主体, 是学习情况的最终反映者, 学生的学习感受, 是教育改革最重要的借鉴。因此, 在教 学过程中教师应当走进学生, 聆听他们的心声, 听取学生的反馈意见。这样更有利于教学的 展开和教学质量的提升, 还利于学生获得好成绩。其次教师应该及时吸收地理信息, 补充地 理事实, 培养学生捕捉性知识的能力。教师应当多设置一些自主性课题, 鼓励学生大胆讲话, 逐步培养学生的主动探究能力。在同学答错问题时, 多给学生一点考虑的时间或做小小的提 示, 让同学继续作答, 这样可以增强学习的自信心与学习的激情。

4. 4. 分配好课外地理学习时间, 构成课外学习时间的最优化 学好地理不只是要利用好课堂时间, 课外对地理学习的时间投入也是一个问题。针对问卷中 学生出现的课后时间分配不合理的问题, 学生在课余时间应该花合理的时间在地理学习上, 每天都利用一点时间来学习地理, 遇到不懂的问题应该采取相应的措施, 例如学生可以主动 上网查阅资料, 看一些书籍, 丰富地理知识, 私底下可以和同学相互讨论、交流地理问题等, 同时也可以向老师咨询, 寻求解决办法。合理分配学习时间, 优化学习时间, 才能使每个学 科达到均衡发展。

\section{5. 致谢}

本文得到云南省卓越青年教师特殊培养项目（自然地理学）资助。

\section{参考文献}

[1] 伦志珍. 中学生地理学习心理分析 [D]. 曲阜师范大学, 2007.

[2] 陈澄. 地理教学论 $[M]$. 上海教育出版社, 2007.

[3] 杨素君. 大理州白族地区高中生地理学习现状及教学对策的研究 [D]. 云南师范大学, 
2005.

[4] 曹琦. 中学地理教学法 [M]. 北京: 高等教育出版社, 2000

[5] 胡伟健. 高中学生地理学习现状的问卷调查及思考 $[\mathrm{J}]$. 福建地理, 2005, 04: 59-60+63.

[6] 孙尚福. 初中地理学习兴趣的培养 [J]. 甘肃教育, 2003, 1：64.

[7] 谢月美. 激发地理学习兴趣, 增加长智丰富情感 $[\mathrm{J}]$. 福建地理, 2000, 04：61-62+60.

[8] 郡胜新. 地理学习策略 $[\mathrm{J}]$. 基础教育研究, 2001, 03：12-13.

[9] 孙大文. 地理教育学 $[M]$. 浙江: 浙江教育出版社, 1992.

[10］于增元. 中学生地理学习兴趣的现状与提升策略 [D]. 鲁东大学, 2013.

\section{Acknowledgement}

This research was financially supported by Yunnan Excellent Young Teacher Project (natural geography).

\section{References}

[1] Zhizhen Lun. Study on Geography Learning Psychology of Middle-school Students, Qufu Normal University, 2007.

[2] Chen Chen. Geography Teaching Theory, Shanghai Education Press, 2007.

[3] Sujun Yang. Study on Current Situation of Geography Learning of Senior High School Students in Dali. Yunnan Normal University, 2007.

[4] Qi Cao. Geography teaching method in middle school. Higher Education Press. 2000.

[5] Weijian Hu. A Survey and Reflection on the Current Situation of Geography Learning of Senior High School Students. Fujian Geography. 2005. No. 4, pp. 59-60+63.

[6] Shangfu Sun. Junior Middle School Geography Learning Interest Cultivation. Gansu Education, 2003. No. 1, pp. 64.

[7] Yuemei Xie. Stimulate Interest in Learning Geography. Fujian Geography. 2000. No. 4, pp. $61-62+60$.

[8] Shengxin Shao. Geography Learning Strategies. Basic Education Research. 2001. No. 3. pp. 12-13.

[9] Dawen Sun. Geography Education. Zhejiang Education Press, 1992.

[10]Zengyuan Yu. Study on Current Situation and Promotion Strategies of Geography Learning interest of Middle-school Students. Ludong University, 2013.

作者简介: 国素梅 $(1991-)$, 女, 云南会泽县人, 主要研究方向为中学地理教学, E-mai 1 : 1347999343@qq. com。导师: 席武俊 (1979-) , 男, 云南文山市人, 副教授, 主要研究方向 为遥感与地理信息系统应用, E-mai1: absxwj@163.com。 